\title{
POINCARÉ SERIES OF SOME HYPERGRAPH ALGEBRAS
}

\author{
E. EMTANDER, R. FRÖBERG, F. MOHAMMADI and S. MORADI
}

\begin{abstract}
A hypergraph $H=(V, E)$, where $V=\left\{x_{1}, \ldots, x_{n}\right\}$ and $E \subseteq 2^{V}$ defines a hypergraph algebra $R_{H}=k\left[x_{1}, \ldots, x_{n}\right] /\left(x_{i_{1}} \cdots x_{i_{k}} ;\left\{i_{1}, \ldots, i_{k}\right\} \in E\right)$. All our hypergraphs are $d$-uniform, i.e., $\left|e_{i}\right|=d$ for all $e_{i} \in E$. We determine the Poincaré series $P_{R_{H}}(t)=\sum_{i=1}^{\infty} \operatorname{dim}_{k} \operatorname{Tor}_{i}^{R_{H}}(k, k) t^{i}$ for some hypergraphs generalizing lines, cycles, and stars. We finish by calculating the graded Betti numbers and the Poincaré series of the graph algebra of the wheel graph.
\end{abstract}

\section{Introduction}

In [6, Chapter 7] the Betti numbers of the graph algebras of the line graph, the cycle graph, and of the star graph are determined. This is generalized to certain "hyperlines", "hypercycles", and "hyperstars" in [2]. A hypergraph $H=(V, E)$, where $V=\left\{x_{1}, \ldots, x_{n}\right\}$ and $E \subseteq 2^{V}$ defines a hypergraph algebra $R_{H}=k\left[x_{1}, \ldots, x_{n}\right] /\left(x_{i_{1}} \cdots x_{i_{k}} ;\left\{i_{1}, \ldots, i_{k}\right\} \in E\right)$. All our hypergraphs are $d$-uniform, i.e., $\left|e_{i}\right|=d$ for all $e_{i} \in E$. A hyperline is a hypergraph with $n d-(n-1) \alpha$ vertices and $n$ edges $e_{1}, \ldots, e_{n}$, where all edges $e_{1}, \ldots, e_{n}$ have size $d$, and $e_{i} \cap e_{j} \neq \emptyset$ and has size $\alpha$ if and only if $|i-j|=1$, a hypercycle is a hypergraph with $n(d-\alpha)$ vertices and $n$ edges $e_{1}, \ldots, e_{n}$, where all edges have size $d$, and $e_{i} \cap e_{j} \neq \emptyset$ and has size $\alpha$ if and only if $|i-j| \equiv 1(\bmod n)$, and the hyperstar is hypergraph with $n(d-\alpha)$ vertices and $n$ edges $e_{1}, \ldots, e_{n}$, where all edges have size $d$, and for all $i, j\left|e_{i} \cap e_{j}\right|=\left|\bigcap_{i=1}^{n} e_{i}\right|=\alpha>0$. We denote the line hypergraph and its algebra with $L_{n}^{d, \alpha}$, the cycle hypergraph and its algebra with $C_{n}^{d, \alpha}$, and the star hypergraph and its algebra $S_{n}^{d, \alpha}$. Their Betti numbers were determined in [2, Chapter 3] (in the first two cases with the restriction $2 \alpha \leq d)$. In this paper we will determine the Poincaré series for the same algebras. The Poincaré series of a graded $k$-algebra $R=k\left[x_{1}, \ldots, x_{n}\right] / I$ is $P_{R}(t)=\sum_{i=1}^{\infty} \operatorname{dim}_{k} \operatorname{Tor}_{i}^{R}(k, k) t^{i}$. [5] is an excellent source for results on Poincaré series. 


\section{Hypercycles and hyperlines when $d=2 \alpha$}

We start with the case $d=2 \alpha$. If $e_{i}=\left\{v_{i 1}, \ldots, v_{i \alpha}, v_{i 1}^{\prime}, \ldots, v_{i \alpha}^{\prime}\right\}$, where $\left\{v_{i j}^{\prime}\right\} \in e_{i+1}$, we start by factoring out all $v_{i k}-v_{i l}$ and $v_{i k}^{\prime}-v_{i l}^{\prime}$. This is a linear regular sequence of length $(n+1)(\alpha-1)$ for the hyperline and of length $n(\alpha-1)$ for the hypercycle. The results are

$$
L_{n, a}^{\prime}=k\left[x_{1}, \ldots, x_{n+1}\right] /\left(x_{1}^{\alpha} x_{2}^{\alpha}, x_{2}^{\alpha} x_{3}^{\alpha}, \ldots, x_{n}^{\alpha} x_{n+1}^{\alpha}\right)
$$

and

$$
C_{n, a}^{\prime}=k\left[x_{1}, \ldots, x_{n}\right] /\left(x_{1}^{\alpha} x_{2}^{\alpha}, x_{2}^{\alpha} x_{3}^{\alpha}, \ldots, x_{n-1}^{\alpha} x_{n}^{\alpha}, x_{n}^{\alpha} x_{1}^{\alpha}\right)
$$

Then

$$
P_{L_{n}^{2 a, a}}(t)=(1+t)^{(n+1)(\alpha-1)} P_{L_{n, a}^{\prime}}(t)
$$

and

$$
P_{C_{n}^{2 a, a}}(t)=(1+t)^{n(\alpha-1)} P_{C_{n, a}^{\prime}}(t),
$$

[5, Theorem 3.4.2(ii)]. Now $L_{n, a}^{\prime}$ and $C_{n, a}^{\prime}$ obviously have the same (ungraded) Poincaré series as the graph algebras

$$
L_{n}=L_{n}^{2,1}=k\left[x_{1}, \ldots, x_{n+1}\right] /\left(x_{1} x_{2}, x_{2} x_{3}, \ldots, x_{n} x_{n+1}\right)
$$

and

$$
C_{n}=C_{n}^{2,1}=k\left[x_{1}, \ldots, x_{n}\right] /\left(x_{1} x_{2}, x_{2} x_{3}, \ldots, x_{n-1} x_{n}, x_{n} x_{1}\right)
$$

respectively.

For a graded $k$-algebra $\bigoplus_{i=0}^{\infty} R_{i}$, the Hilbert series of $R$ is defines as $H_{R}(t)=\sum_{i=0}^{\infty} \operatorname{dim}_{k}\left(R_{i}\right) t^{i}$. The exact sequences

$$
0 \longrightarrow\left(x_{n+1}\right) \longrightarrow L_{n} \stackrel{x_{n+1} \cdot}{\longrightarrow} L_{n} \longrightarrow L_{n} /\left(x_{n+1}\right) \longrightarrow 0
$$

and

$$
0 \longrightarrow\left(x_{n+1}\right) \longrightarrow L_{n} \longrightarrow L_{n} /\left(x_{n+1}\right) \longrightarrow 0
$$

and $L_{n} /\left(x_{n+1}\right) \simeq L_{n-1}$ and $\left(x_{n+1}\right) \simeq L_{n-2} \otimes k[x]$ gives

$$
H_{L_{n}}(t)=H_{L_{n-1}}(t)+\frac{t}{1-t} H_{L_{n-2}}(t) .
$$

The exact sequences

$$
0 \longrightarrow\left(x_{1}, x_{n-1}\right) \longrightarrow C_{n} \stackrel{x_{n} \cdot}{\longrightarrow} C_{n} \longrightarrow L_{n-2} \longrightarrow 0
$$

and

$$
0 \longrightarrow\left(x_{1}, x_{n-1}\right) \longrightarrow C_{n} \longrightarrow C_{n} /\left(x_{1}, x_{n-1}\right) \longrightarrow 0
$$


and $C_{n} /\left(x_{1}, x_{n-1}\right) \simeq L_{n-4} \otimes k[x]$ gives

$$
H_{C_{n}}(t)=H_{L_{n-2}}(t)-\frac{t}{(1-t)} H_{L_{n-4}}(t) .
$$

Now $C_{n}$ and $L_{n}$ are (as all graph algebras) Koszul algebras [3, Corollary 2], so $P_{C_{n}}(t)=1 / H_{C_{n}}(-t)$ and $P_{L_{n}}(t)=1 / H_{L_{n}}(-t)$. Since $L_{0}=k\left[x_{1}\right]$ and $L_{1}=$ $k\left[x_{1}, x_{2}\right] /\left(x_{1} x_{2}\right)$, we have $H_{L_{0}}(t)=1 /(1-t)$ and $H_{L_{1}}(t)=(1+t) /(1-t)$.

We give the first Hilbert series:

$$
\begin{aligned}
& H_{L_{2}}(t)=\left(1+t-t^{2}\right) /(1-t)^{2}, \quad H_{L_{3}}(t)=(1+2 t) /(1-t)^{2}, \\
& H_{L_{4}}(t)=\left(1+2 t-t^{2}-t^{3}\right) /(1-t)^{3}, \quad H_{L_{5}}(t)=\left(1+3 t+t^{2}-t^{3}\right) /(1-t)^{3}, \\
& H_{C_{3}}(t)=(1+2 t) /(1-t), \quad H_{C_{4}}(t)=\left(1+2 t-t^{2}\right) /(1-t)^{2}, \\
& H_{C_{5}}(t)=\left(1+3 t+t^{2}\right) /(1-t)^{3}, \quad H_{C_{6}}(t)=\left(1+3 t-2 t^{3}\right) /(1-t)^{3} .
\end{aligned}
$$

Thus we get

$$
\begin{aligned}
& P_{L_{2}}(t)=(1+t)^{2} /\left(1-t-t^{2}\right), \quad P_{L_{3}}(t)=(1+t)^{2} /(1-2 t), \\
& P_{L_{4}}(t)=(1+t)^{3} /\left(1-2 t-t^{2}+t^{3}\right), \quad P_{L_{5}}(t)=(1+t)^{3} /\left(1-3 t+t^{2}+t^{3}\right), \\
& P_{C_{3}}(t)=(1+t) /(1-2 t), \quad P_{C_{4}}(t)=(1+t)^{2} /\left(1-2 t-t^{2}\right), \\
& P_{C_{5}}(t)=(1+t)^{2} /\left(1-3 t+t^{2}\right), \quad P_{C_{6}}(t)=(1+t)^{3} /\left(1-3 t+2 t^{3}\right) .
\end{aligned}
$$

We collect the results in

THeOREM 2.1. The Poincaré series of $L_{n}$ and $C_{n}$ satisfy the recursion formulas

$$
P_{L_{n}}(t)=\frac{(1+t) P_{L_{n-1}}(t) P_{L_{n-2}}(t)}{(1+t) P_{L_{n-2}}(t)-t P_{L_{n-1}}(t)}
$$

where $P_{L_{0}}(t)=1+t$ and $P_{L_{1}}(t)=(1+t) /(1-t)$ and

$$
P_{C_{n}}(t)=\frac{(1+t) P_{L_{n-2}}(t) P_{L_{n-4}}(t)}{P_{L_{n-2}}(t)+(1+t) P_{L_{n-4}}(t)} .
$$

Furthermore

$$
P_{L_{n}^{2 \alpha, \alpha}}(t)=(1+t)^{(n+1)(\alpha-1)} P_{L_{n}}(t)
$$

and

$$
P_{C_{n}^{2 \alpha, \alpha}}(t)=(1+t)^{n(\alpha-1)} P_{C_{n}}(t)
$$

\section{Hypercycles and hyperlines when $2 \alpha<d$}

Next we turn to the case $2 \alpha<d$. Now each edge has a free vertex, i.e. a vertex which does not belong to any other edge. Then the Taylor resolution (cf e.g. [4]) is minimal. In that case there is a formula for the Poincaré 
series in terms of the graded homology of the Koszul complex [4, Corollary to Proposition 2]. Let $R$ be a monomial ring for which the Taylor resolution is minimal. Then the homology of the Koszul complex $H\left(K_{R}\right)$ is of the form $H\left(K_{R}\right)=k\left[u_{1}, \ldots, u_{N}\right] / I$, where $I$ is generated by a set of monomials of degree 2 . Define a bigrading induced by $\operatorname{deg}\left(u_{i}\right)=\left(1,\left|u_{i}\right|\right)$, where $\left|u_{i}\right|$ is the homological degree. Then $P_{R}(t)=(1+t)^{e} / H_{R}(-t, t)$, where $e$ is the embedding dimension and $H_{R}(x, y)$ is the bigraded Hilbert series of $H\left(K_{R}\right)$, see [4].

We begin with the hypercycle. The homology of the Koszul complex (which computes the Betti numbers) is generated by $\left\{z_{I}\right\}$, where $I=\{i, i+1, \ldots, j\}$ corresponds to a path $\left\{e_{i}, e_{i+1}, \ldots, e_{j}\right\}$ in $C_{n}^{d, \alpha}$ (indices counted $\left.(\bmod n)\right)$. Thus there are $n$ generators in all homological degrees $<n$ and one generator in homological degree $n$. We have $z_{I} z_{J}=0$ if $I \cap J \neq \emptyset$. Thus the surviving monomials are of the form $m=z_{I_{1}} \cdots z_{I_{r}}$, where $I_{i} \cap I_{j}=\varnothing$ if $i \neq j$. The bidegree of $m$ is $\left(r, \sum_{j=1}^{r}\left|I_{j}\right|\right)$. Let $\sum_{j=1}^{r}\left|I_{j}\right|=i$. Then $m$ lies in $H(K)_{i, d i-(i-r) \alpha}$. The graded Betti numbers are determined in [2, Chapter 3]. The nonzero Betti numbers are $\beta_{i, d i-(i-r) \alpha}=\frac{n}{r}\left(\begin{array}{c}i-1 \\ r-1\end{array}\right)\left(\begin{array}{c}n-i-1 \\ r-1\end{array}\right)$ if $1 \leq r \leq i<n$ and $\beta_{n, n(d-\alpha)}=1$. (As usual $\left(\begin{array}{l}a \\ b\end{array}\right)=0$ if $b>a$.) This gives the Poincaré series.

Next we consider the hyperline. The homology of the Koszul complex is generated by $\left\{z_{I}\right\}$, where $I=\{i, i+1, \ldots, j\}$ corresponds to a path $\left\{e_{i}, e_{i+1}, \ldots, e_{j}\right\}$ in $L(n, d, \alpha)$. Thus there are $n+1-i$ generators of homological degree $i$. We have $z_{I} z_{J}=0$ if $I \cap J \neq \varnothing$. The graded Betti numbers are determined in [2, Chapter 3]. The nonzero Betti numbers are $\beta_{i, d i-(i-r) \alpha}=\left(\begin{array}{c}i-1 \\ r-1\end{array}\right)\left(\begin{array}{c}n-i+1 \\ r\end{array}\right)$ if $1 \leq r \leq i \leq n$. The same reasoning as above gives the Poincaré series. We state the results in a theorem.

THEOREM 3.1. If $2 \alpha<d$, then

$$
P_{C_{n}}(t)=\frac{(1+t)^{n(d-\alpha)}}{1+\sum_{1 \leq r \leq i<n}(-1)^{r} \frac{n}{r}\left(\begin{array}{c}
i-1 \\
r-1
\end{array}\right)\left(\begin{array}{c}
n-i-1 \\
r-1
\end{array}\right) t^{i+r}-t^{n+1}},
$$

and

$$
P_{L_{n}}(t)=\frac{(1+t)^{n(d-\alpha)+\alpha}}{1+\sum_{1 \leq r \leq i \leq n}(-1)^{r}\left(\begin{array}{c}
i-1 \\
r-1
\end{array}\right)\left(\begin{array}{c}
n-i+1 \\
r
\end{array}\right) t^{i+r}} .
$$

\section{The hyperstar}

We conclude with a hypergraph generalizing the star graph. Suppose $\left|e_{i}\right|=d$ for all $i, 1 \leq i \leq n$, and that if $i \neq j$, then $\left|e_{i} \cap e_{j}\right|=\left|\bigcap_{i=1}^{n} e_{i}\right|=\alpha<d$. Then the ideal is of the form $m\left(m_{1}, \ldots, m_{n}\right)$, where $m$ is a monomial of degree $\alpha$. Then the hypergraph ring $S_{n}^{d, \alpha}$ is Golod [5, Theorem 4.3.2]. This means that 
THEOREM 4.1.

$$
P_{S_{n}^{d, \alpha}}(t)=(1+t)^{|V|} /\left(1-\sum \beta_{i} t^{i+1}\right)=(1+t)^{n(d-\alpha)+\alpha} /\left(1-\sum\left(\begin{array}{l}
n \\
i
\end{array}\right) t^{i+1}\right) .
$$

\section{The wheel graph}

Finally we consider the wheel graph $W_{n}$, which is $C_{n}$ with an extra vertex (the center) which is connected to all vertices in $C_{n}$. We let $W_{n}$ also denote the graph algebra $k\left[x_{0}, \ldots, x_{n}\right] /\left(x_{1} x_{2}, x_{2} x_{3}, \ldots, x_{n} x_{1}, x_{0} x_{1}, \ldots, x_{0} x_{n}\right)$.

TheOREM 5.1. Let $W_{n}$ be a wheel graph on $n+1$ vertices. Then the Betti numbers of $W_{n}$ are as follows:

(i) If $j>i+1$, then $\beta_{i, j}\left(k\left[\Delta_{W_{n}}\right]\right)=\beta_{i, j}\left(C_{n}\right)+\beta_{i-1, j-1}\left(C_{n}\right)$.

(ii) If $j=i+1$, then $\beta_{i, i+1}\left(W_{n}\right)=\beta_{i, i+1}\left(C_{n}\right)+\beta_{i-1, i}\left(C_{n}\right)+\left(\begin{array}{c}n \\ i\end{array}\right)$.

Proof. Assume that $V\left(W_{n}\right)=\left\{x_{0}, x_{1}, \ldots, x_{n}\right\}$ and $C_{n}=W_{n} \backslash\left\{x_{0}\right\}$. It is easy to see that $\Delta_{W_{n}}=\Delta_{C_{n}} \cup\left\{x_{0}\right\}$, where $\Delta_{W_{n}}$ and $\Delta_{C_{n}}$ are the independence complexes of $W_{n}$ and $C_{n}$. It implies that for any $i \geq 1, H_{i}\left(\Delta_{W_{n}}\right)=H_{i}\left(\Delta_{C_{n}}\right)$. Thus, if $j>i+1$, from Hochster's formula ([1, Theorem 5.5.1]) and the observation above one has the result. Now assume that $j=i+1$. Then

$$
\begin{aligned}
\beta_{i, i+1}\left(W_{n}\right) & =\sum_{S \subseteq V\left(W_{n}\right),|S|=i+1} \operatorname{dim}\left(\tilde{H}_{0}\left(\Delta_{S}\right)\right) \\
& =\sum_{S \subseteq V\left(C_{n}\right),|S|=i+1} \operatorname{dim}\left(\tilde{H}_{0}\left(\Delta_{S}\right)\right)+\sum_{S \subseteq V\left(W_{n}\right), S=S^{\prime} \cup\left\{x_{0}\right\}} \operatorname{dim}\left(\tilde{H}_{0}\left(\Delta_{S}\right)\right) .
\end{aligned}
$$

For any $S \subseteq V\left(W_{n}\right)$ and $S_{0} \subseteq V\left(C_{n}\right)$, let $r_{S}$ and $r_{S_{0}}^{\prime}$ denotes the number of connected components of $\Delta_{S}$ in $V\left(W_{n}\right)$ and $\Delta_{S_{0}}$ in $V\left(C_{n}\right)$ respectively. Then we have $\sum_{S \subseteq V\left(W_{n}\right), S=S_{0} \cup\left\{x_{0}\right\}} \operatorname{dim}\left(\widetilde{H}_{0}\left(\Delta_{S}\right)\right)=\sum_{S \subseteq V\left(W_{n}\right), S=S_{0} \cup\left\{x_{0}\right\}}\left(r_{S}-1\right)$. For any $S \subseteq V\left(W_{n}\right)$ such that $S=S_{0} \cup\left\{x_{0}\right\}$, we have $r_{S}=r_{S_{0}}^{\prime}+1$. Therefore

$$
\begin{aligned}
\sum_{S \subseteq V\left(W_{n}\right), S=S_{0} \cup\left\{x_{0}\right\}} \operatorname{dim}\left(\widetilde{H}_{0}\left(\Delta_{S}\right)\right) & =\sum_{S_{0} \subseteq V\left(C_{n}\right),\left|S_{0}\right|=i} \operatorname{dim}\left(\widetilde{H}_{0}\left(\Delta_{S_{0}}\right)\right)+\left(\begin{array}{c}
n \\
i
\end{array}\right) \\
& =\beta_{i-1, i}\left(C_{n}\right)+\left(\begin{array}{c}
n \\
i
\end{array}\right) .
\end{aligned}
$$

The term $\left(\begin{array}{l}n \\ i\end{array}\right)$ is the number of subsets $S_{0}$ of $V\left(C_{n}\right)$ of cardinality $i$.

Substituting the $\beta_{i, j}\left(C_{n}\right)$ from of [6, Theorem 7.6.28] we have the following corollary. 
Corollary 5.2. Let $W_{n}$ be the wheel graph on $n+1$ vertices. Then the Betti numbers of $W_{n}$ are as follows:

(i) If $n=3$, then $\beta_{2,3}\left(W_{3}\right)=8, \beta_{3,4}\left(W_{3}\right)=3$. If $n=4$, then $\beta_{3,4}\left(W_{4}\right)=9$, $\beta_{4,5}\left(W_{4}\right)=2$. Otherwise $\beta_{i, i+1}\left(W_{n}\right)=n\left(\begin{array}{c}2 \\ i-1\end{array}\right)+\left(\begin{array}{c}n \\ i\end{array}\right)$.

(ii) If $n=3 m$, then $\beta_{2 m, n}\left(W_{n}\right)=3 m+2, \beta_{2 m+1, n+1}\left(W_{n}\right)=2$. If $n=3 m+1$, then $\beta_{2 m+1, n}\left(W_{n}\right)=3 m+2, \beta_{2 m+2, n+1}\left(W_{n}\right)=1$. If $n=3 m+2$, then $\beta_{2 m, n}\left(W_{n}\right)=\beta_{2 m+1, n+1}\left(W_{n}\right)=1$. Otherwise, if $j>i+1$, then $\beta_{i, j}\left(W_{n}\right)=\frac{n}{n-2(j-i)}\left(\begin{array}{c}n-2(j-i) \\ j-i\end{array}\right)\left(\begin{array}{c}j-i-1 \\ 2 i-j\end{array}\right)$.

We can also determine the Poincaré series for the wheel graph algebra. This is also a Koszul algebra, and $H_{W_{n}}(t)=H_{C_{n}}(t)+t /(1-t)$. Since $P_{W_{n}}(t)=$ $1 / H_{W_{n}}(-t)$ and $P_{C_{n}}(t)=1 / H_{C_{n}}(-t)$, this gives

THEOREM 5.3.

$$
P_{W_{n}}(t)=\frac{P_{C_{n}}(t)(1+t)}{1+t-t P_{C_{n}}(t)}
$$

\section{REFERENCES}

1. Bruns, W., Herzog, J., Cohen-Macaulay Rings, revised ed., Cambridge Studies in Adv. Math. 39, Cambridge Univ. Press, Cambridge 1998.

2. Emtander, E, Mohammadi, F., Moradi, S., Some algebraic properties of hypergraphs, Czecholovak Math. J. 61 (2011), 577-607.

3. Fröberg, R., Determination of a class of Poincaré series, Math. Scand. 37 (1975), 29-39.

4. Fröberg, R., Some complex constructions with applications to Poincaré series, pp. 272-284 in: Séminaire d'Algèbre Paul Dubreil, Proc. Paris 1977-78, Lecture Notes in Math. 740, Springer, Berlin 1979.

5. Gulliksen, T. H., Levin, G., Homology of local rings, Queen's Paper in Pure and Appl. Math. 20, Queen's Univ., Kingston, Ont. 1969.

6. Jacques, S., Betti Numbers of Graph Ideals, Ph.D. thesis, Univ. of Sheffield (2004), arXiv: math/0410107.

DEPARTMENT OF MATHEMATICS

STOCKHOLM UNIVERSITY

SE 10691 STOCKHOLM

SWEDEN

E-mail: erice@math.su.se ralff@math.su.se
DEPARTMENT OF MATHEMATICS AND COMPUTER SCIENCE AMIRKABIR UNIVERSITY OF TECHNOLOGY TEHRAN

IRAN

E-mail: f_mohammadi@aut.ac.ir s_moradi@aut.ac.ir 